\title{
Psychiatric screening for migraine patients
}

\author{
O. Gambini • B. Biagianti - L. Grazzi • \\ S. Usai $\cdot$ S. Scarone $\cdot$ G. Bussone
}

(C) Springer-Verlag Italia 2013

\begin{abstract}
Psychiatric disorders in migraine patients have a higher prevalence than general population. The presence of psychiatric comorbidities may influence the complexity of the migraine pictures and be related to medication overuse. Severely impaired chronic migraineurs presenting with medication overuse are a challenge for headache clinics. Psychiatric comorbities, such as dependency-like behaviors, anxiety and mood symptoms, might account for headacherelated disability and recurrent relapses into medication overuse after a successful detoxification. Within a sample of 63 chronic migraineurs with medication overuse and severe disability, we investigated to which extent clinical severity, affective states and attitudes about medication impact the overall functioning at time of detoxification. To unravel whether some of these factors could predict their long-term outcome, we followed and retest them 1 year after withdrawal. We hypothesized that the detoxification would have led to a partial improvement and not modified the attitudes
\end{abstract}

O. Gambini $(\bowtie) \cdot$ B. Biagianti $\cdot$ S. Scarone

Dipartimento di Scienze della Salute, Università degli Studi

di Milano - AO San Paolo, Via A. di Rudinì 8/A,

20142 Milan, Italy

e-mail: orsola.gambini@unimi.it

B. Biagianti

Biomagnatic Imaging Laboratory, Department of Radiology,

University of California, San Francisco, San Francisco,

CA 94143, USA

B. Biagianti

Department of Psychiatry, San Francisco Department

of Veterans Affairs Medical Center, San Francisco,

CA 94121, USA

L. Grazzi · S. Usai · G. Bussone

Headache Unit, Department of Clinical Neuroscience,

C. Besta Neurological Institute and Foundation, Milan, Italy toward medication and dependence. Detoxification improves most of the clinical and affective measures, but does not free from significant levels of pain intensity and headache-related disability. The partial benefit from detoxification, the severity bias and the maladaptive cognitive profile led us to believe that subgroups of chronic-relapsing migraineurs deserve a multidisciplinary approach that addresses not only the reduction of clinical severity but also specific cognitive and behavioral impairments.

Keywords Migraine - Medication overuse .

Psychiatric comorbidities

\section{Introduction}

Chronic migraine (CM) with Medication overuse (MO) affects upto $15-30 \%$ of patients with headaches referring to tertiary care clinics [1-3]. Some of them present dependency-like behaviors with medications, despite being aware of and concerned about their negative consequences, i.e. compromised social functioning and severe impact on the quality of life [4, 5]. The underlying neurobiology of $\mathrm{MO}$ is not well understood. Although it might come from coping with pain and disability associated with migraine, a persistent dysfunction of the orbitofrontal cortex (OFC) and the relative decision-making deficit have been recently reported [6-8]. These deficits might explain the above-mentioned maladaptive behaviors and place $\mathrm{MO}$ in the substance use disorders spectrum [6].

Detoxification from the offending drug is the first line treatment $[9,10]$. However, long-term studies indicate that a high proportion of patients relapse into MO within months or years, even after a successful detoxification [11, 12]. Reasons for relapses might go beyond the frequency and the intensity of headaches. 
First, the comorbidity with anxiety and mood disorders has been associated with a negative long-term prognosis in migraine and might play a role in perpetuating MO [13]. A bidirectional influence has been observed between migraine and depression, with each disorder increasing the risk for onset of the other $[14,15]$. Second, the severity of dependency-like behaviors and the excessive confidence in the healing power of medications could jeopardize the benefit of detoxification and predispose migraneurs to persist in overusing medications [16-18].

Several studies suggest predictors of relapse after a successful detoxification in samples of chronic migraineurs with MO [1, 12, 19-22]. However, in tertiary centers, these putative factors are present and severe to such an extent that they could reciprocally influence each other and blur the clinical presentation and the physician manageability.

The aim of this study was to pick a sample of severely impaired chronic migraineurs with MO and study whether some of the predictors described by literature could be identified as risk factors for relapse. We hypothesized that detoxification as part of treatments for chronic migraine with MO would have led only to a partial improvement. To this purpose, we first delineated how psychopathological features and attitudes toward medication interact with clinical features. One year after detoxification, chronic migraineurs were reevaluated, in order to unravel whether their long-term outcome could be predicted by their baseline migraine characteristic, baseline affective states and their attitudes about medication and dependence.

\section{Methods}

\section{Participants}

Sixty-three patients with $\mathrm{CM}$ and $\mathrm{MO}$ were recruited at the Headache Center of the Foundation IRCCS Neurological Institute Carlo Besta in Milan, whose Ethics Committee approved the study.

$\mathrm{CM}$ (i.e. migraine headaches present on $\geq 15$ days in a month on a regular basis for $>3$ months) and MO (intake of simple analgesic on $\geq 15$ days/month or of any combination of ergotamine, triptans, analgesics, and/or opioids on $\geq$ days per month on a regular basis for $>3$ months) were diagnosed according to the current criteria of the International Headache Society [23] Exclusion criteria were other chronic pain conditions, pregnancy, progressive neurological disorders, present or past psychotic disorders, present or past substances and alcohol use disorders, use of antipsychotic drugs in the last 6 months. All patients were informed of the setting-up of the observatory database and signed a written consent before participating.
After enrollment, patients underwent detoxification in a 5-day Day Hospital regimen and a neuropsychiatric evaluation by means of structured and semi-structured instruments. They were encouraged to keep headaches and medications record and taught how to manage migraine episodes with NSAIDs and triptans. When needed, after withdrawal, prophylaxis therapy was prescribed. After 1 year, they underwent the same evaluation in an outpatient regimen, and were classified into two groups: those who ceased MO and those who relapsed into MO.

\section{Measures}

All subjects were evaluated by means of the rating scales listed below.

The Hamilton Rating Scales for Anxiety and Depression $[24,25]$ for the measurement of intensity of depression and anxiety symptoms. The Migraine Disability Assessment Questionnaire (MIDAS) [26, 27] which measures headache-related disability in the past 3 months by means of five questions about work, household and social-related disability, the frequency of headaches and the intensity of headache pain (on an 11-point scale, ranging from 0 to 10). The Severity of Dependence Scale (SDS) [28], originally created to investigate opiate dependence, used to predict patterns of MO and dependency-like behaviors among chronic headache patients $[17,18]$. The higher the total score (range 0-15), the higher the level of dependence. The Symptom Checklist-90-Revised (SCL-90-R) Self-Report [29] Last-month is a self-report symptom inventory used in medical or psychiatric conditions. In the study, we used the Global Severity Index (GSI) score, which summarizes the overall psychopathological impairment.

Statistical analysis

All statistical analyses were performed using SPSS 17.0 for Windows (SPSS Inc, Chicago, IL, USA) and R2.8.1 (R Development Core Team, 2006). First, correlational analyses were employed to explore possible relations at baseline between clinical features, affective measures and attitudes toward medication and dependence. Second, a series of $t$ test for paired samples was used to assess the evolution of psychological and clinical features 1 year after the detoxification. A further series of $t$ test for independent samples and Chi-squared tests were performed to investigate the differences 1 year after detoxification in all the studied domains between those who ceased MO and those who relapsed into it. With the aim of clarifying the value of single variables in determining the relapse, the disability and the persistent analgesic intake after detoxification, we ran a series of bivariate analyses whose outcome variables 
were, respectively, the presence of relapse after 12 months, the number of tablets per month and the MIDAS total score. Explanatory variables taken from baseline evaluation were gender, age, years with migraine, years with medication overuse, frequency of headache per month, number of tablets per month, intensity of headache pain, MIDAS total score, past history of psychiatric disorder, psychiatric diagnosis at time of evaluation, GSI at SCL90R questionnaire, scores at Hamilton Rating Scales for Anxiety and Depression and SDS score.

The normality assumption was assessed using the Kolmogorov-Smirnov test, with Lilliefors significance correction. For bivariate analysis, Kruskal-Wallis rank sum test and Fisher's two-tailed exact test were performed to, respectively, analyze numerical and categorical variables. Due to the lack of significance in Levene's test, we decided to report data as expressed by mean and standard deviation. In consideration of the exploratory nature of the study, we referred to levels of significance of $p$ value $<0.05$. All tests were two-tailed.

\section{Results}

Demographic and clinical characteristics of CM patients with $\mathrm{MO}$ at baseline are below depicted. The population consisted of 63 patients ( $81 \%$ female), aged between 23 and 71 years (mean age $=42.1, \mathrm{SD}=10.2$ ). The preexisting primary headache subtype was migraine without aura.

Most active constituents for migraine acute treatment were simple analgesics, triptans and low doses of opiates in combination with non-opiate analgesics. 21 patients fulfilled ICHD criteria for triptan-overuse headache, 33 those for of NSAID overuse headache. The other nine patients could not be subclassified as they used at least both classes of medication. An analysis of variance was performed on the basis of different symptomatic medications used for migraine attack. We found no significant differences in the clinical and psychological studied domains.

Different preventive medications, single or in combination, were or had been used in the sample according to patients' clinical status (valproic acid, topiramate, betablockers, amitriptyline, flunarizine). At baseline, 28 patients were off preventive treatment before due to lack of efficacy while 35 were under single compound or combination prophylaxis. After detox, 31 patients were given prophylaxis to control possible attacks. An on-off prophylaxis split analysis was performed, notwithstanding the type and the amount of different medications. From the series of $t$ tests for independent samples, no difference emerged across the clinical, psychological and affective and measures.
Our sample was characterized by a long duration of migraine history (mean years with migraine $=23.34$, $\mathrm{SD}=13.08$, minimum 1 year, maximum 60 years) of medication overuse (mean years with overuse $=2.76$, $\mathrm{SD}=5.96$, minimum 3 months, maximum 36 years). The intensity of headache pain on VAS (mean $=7.71$, $\mathrm{SD}=1.61$ ), the frequency of headaches (mean episodes per month $=22.19, \mathrm{SD}=6.54)$, and medication intake (mean number of tablets per month $=32.95, \mathrm{SD}=23.21$ ) accounted for the high very high rates headache-related disability (mean MIDAS score was 68.87 with a 49.86 SD) and severe dependency-like behaviors (mean SDS score was 8.67 with a $2.19 \mathrm{SD}$; cut off for medication dependence is 5).

Twenty-four subjects $(38.7 \%)$ had a past history of mood or anxiety disorder; after Axis 1 clinical evaluation 18 patients $(29 \%)$ were, respectively, diagnosed with an anxiety or depressive disorder. No significant differences in the clinical and psychological studied features were found between those who had or had had a psychiatric disorder and those who did not.

Correlational analysis (Table 1) revealed that all the measured clinical variables (years with migraine, years with medication overuse, frequency of headache per month, number of tablets per month, intensity of headache pain) went through reciprocal influences that contribute as independent factors in determining the headache-related disability as measured by MIDAS.

After 1 year of follow-up, 21 out of the 63 patients who originally underwent the detoxification dropped out (33\%): 4 fell into the exclusion criteria: 2 for suicide attempt and 2 for pregnancy, 10 referred to other headache centers, 4 did not came for follow-up visit; 3 patients relapsed into MO within a month after detoxification and were, therefore, excluded from longitudinal analyses. No significant differences in baseline characteristics were found between dropouts and those who completed study follow-up. Data for longitudinal statistical inferences are indeed available for 42 patients: according to ICHD-II criteria, 31 of them ceased MO and restored an episodic migraine pattern while 11 patients, in spite of the temporary resolution of $\mathrm{MO}$, relapsed into $\mathrm{MO}$ within 1 year after detoxification. Considering the follow-up sample regardless of relapse (Table 2a), subjects showed significant decreases in most clinical and psychological tested domains (frequency of headaches per month, number of tablets per month, intensity of headache pain, MIDAS total score, GSI at SCL-90R questionnaire, scores at Hamilton Rating Scale for Depression, SDS score). These findings highlight the benefit of detoxification. A comparative $t$ test analysis for independent samples between those with positive and negative outcomes (Table $2 b$ ) showed expected differences in some clinical measurements, such as the 
Table 1 Bivariate statistical analysis with Pearson's productmoment correlation among clinical measurements at baseline

\begin{tabular}{|c|c|c|c|}
\hline \multicolumn{4}{|l|}{ Correlations } \\
\hline $\begin{array}{l}\text { Years with } \\
\text { medication } \\
\text { overuse }\end{array}$ & $\begin{array}{l}\text { Frequency } \\
\text { of } \\
\text { headaches } \\
\text { per month }\end{array}$ & $\begin{array}{l}\text { Number } \\
\text { of tablets } \\
\text { per } \\
\text { month }\end{array}$ & $\begin{array}{l}\text { Intensity } \\
\text { of } \\
\text { headache } \\
\text { pain on } \\
\text { VAS }\end{array}$ \\
\hline
\end{tabular}

\begin{tabular}{|c|c|c|c|c|}
\hline \multicolumn{5}{|c|}{ Years with migraine } \\
\hline $\begin{array}{l}\text { Pearson } \\
\text { correlation }\end{array}$ & $0.329 * *$ & 0.157 & $0.283 *$ & -0.036 \\
\hline Sig. (2-tailed) & 0.008 & 0.220 & 0.025 & 0.780 \\
\hline \multicolumn{5}{|c|}{ Years with medication overuse } \\
\hline $\begin{array}{l}\text { Pearson } \\
\text { correlation }\end{array}$ & 1.000 & 0.173 & 0.456 & 0.207 \\
\hline Sig. (2-tailed) & & 0.176 & 0.000 & 0.103 \\
\hline \multicolumn{5}{|c|}{ Frequency of headaches per month } \\
\hline $\begin{array}{l}\text { Pearson } \\
\text { correlation }\end{array}$ & 0.173 & 1.000 & 0.375 & 0.203 \\
\hline Sig. (2-tailed) & 0.176 & & 0.002 & 0.111 \\
\hline \multicolumn{5}{|c|}{ Number of tablets per month } \\
\hline $\begin{array}{l}\text { Pearson } \\
\text { correlation }\end{array}$ & $0.456 * *$ & $0.375 * *$ & 1.000 & 0.341 \\
\hline Sig. (2-tailed) & 0.000 & 0.002 & & 0.006 \\
\hline \multicolumn{5}{|c|}{ Intensity of headache pain on VAS } \\
\hline $\begin{array}{l}\text { Pearson } \\
\text { correlation }\end{array}$ & 0.207 & 0.203 & $0.341 * *$ & 1.000 \\
\hline Sig. (2-tailed) & 0.103 & 0.111 & 0.006 & \\
\hline \multicolumn{5}{|c|}{ MIDAS total score } \\
\hline $\begin{array}{l}\text { Pearson } \\
\text { correlation }\end{array}$ & 0.203 & $0.311^{*}$ & 0.219 & $\mathbf{0 . 3 7 0} * *$ \\
\hline Sig. (2-tailed) & 0.111 & 0.013 & 0.085 & 0.003 \\
\hline
\end{tabular}

* Correlation is significant at the 0.05 level (2-tailed)

** Correlation is significant at the 0.01 level (2-tailed)

tablets intake and headaches frequencies. Patients who relapsed into MO were more anxious $(15.55 \pm 8.13$ vs. $10.19 \pm 5.86)$ with maladaptive dependency-like behaviors (powerlessness, medications unmanageability, loss of control over medications despite the adverse consequences over headache). Mean SDS score in the negative outcome subgroup was unmodified compared to baseline $(9.36 \pm 2.06)$.

A series of bivariate analyses was performed to identify risk factors for long-term relapse, headacherelated disability and medication intake. Three separate analyses were conducted as preliminary inspections propaedeutic for running a subsequent multivariate regression analysis to detect predictors. However, the scant sample numerosity would not have allowed any inference for a putative multivariate regression model. In addition, no explanatory variables were found for any of selected outcomes.

\section{Discussion}

The identification of relapse predictors is still an unachieved target in chronic migraine with medication overuse.

The results from 1-year and 4-year follow-up studies are currently inconsistent. Many researchers pointed out the severity of baseline migraine as source of the main outcome predictors [12, 20]. However, long-term prospective studies were not equally encouraging, indicating that few or none of the baseline characteristics significantly influenced the outcome measures [11]. Anxiety and mood symptoms have been considered capable of influencing the long-term outcome. In their 4-year follow-up study, Hagen and colleagues [21] reported that a low depression score at baseline was the only factor associated with a favorable outcome. As Table 2a shows, our sample was affected by severe chronic migraine and $\mathrm{MO}$ characterized by severe dependency-like behaviors, significant anxiety and depression levels.

Pearson analysis showed that the higher is the medication intake, the longer are the history of MO, the frequency of migraine episodes and the intensity of headache pain. Furthermore, the longer is the history of migraine, the higher are the medication intake and the duration of MO. Therefore, the headache-related disability directly results from the frequency and the intensity of migraine episodes. Most studied variables have reciprocal influences that blurred the clinical presentation to the degree that it was no longer possible to determine the single role of each variable in contributing to the overall impairment and optimize individual-tailored treatment. The severity of our sample is confirmed by the symptomatic and prophylactic polytherapy the patients were kept on. On-off split analyses were performed on the basis of different symptomatic and prophylactic medications for clinical, psychological and affective measures. The lack of significant differences might derive from the subcategories' overlap, given the great variability of medication protocols in comparison with the small size of the sample.

We selected the presence of relapse, the headacherelated disability and the number of tablets per month as the three major indicators of positive outcome after 1 year. Neither the type of medication overused nor the presence of a prophylactic therapy predicted any of three selected outcomes. Again, the complexity of therapeutic protocols might account for the lack of significance in results. Beyond the evidence that the follow-up sample showed significant improvements in most of the clinical and affective measures regardless of relapse, the improvement of clinical, affective and dependence symptoms results partial.

In this study, we tried to delineate the features of a sample of highly impaired chronic migraineurs with MO. 
Table 2 Student $t$ test for paired samples (baseline vs. one year follow-up) and Student $t$ test for independent samples (negative outcome vs. positive outcome)

\begin{tabular}{|c|c|c|c|c|c|c|}
\hline & \multicolumn{3}{|c|}{ (a) Paired samples } & \multicolumn{3}{|c|}{ (b) Independent samples } \\
\hline & $\begin{array}{l}\text { Baseline } \\
(N=43)\end{array}$ & $\begin{array}{l}\text { 1-year follow-up } \\
(N=43)\end{array}$ & $P$ & $\begin{array}{l}\text { Positive outcome } \\
\text { At } 1 \text {-year follow-up } \\
(N=31)\end{array}$ & $\begin{array}{l}\text { Negative outcome } \\
\text { At } 1 \text {-year follow-up } \\
(N=11)\end{array}$ & $P$ \\
\hline Frequency of headaches per month* & $21.74(6.41)$ & $11.19(7.37)$ & $0.000 * *$ & $8.32(5.46)$ & $19.27(5.99)$ & $0.000 * *$ \\
\hline Number of tablets per month* & 30.05 (21.93) & $15.04(16.60)$ & $0.000 * *$ & $7.80(4.41)$ & $35.45(21.23)$ & $0.000 * *$ \\
\hline Intensity of headache pain on VAS* & $7.55(1.71)$ & $6.52(1.70)$ & $0.001 * *$ & $6.32(1.72)$ & $7.09(1.58)$ & 0.202 \\
\hline MIDAS total score* & $68.83(44.97)$ & $38.64(38.90)$ & $0.005 * *$ & $30.32(26.13)$ & $62.09(57.76)$ & 0.105 \\
\hline HAM-A score* & $12.60(7.14)$ & $11.60(6.85)$ & 0.402 & $10.19(5.87)$ & $15.55(8.13)$ & $0.024 * *$ \\
\hline HAM-D score* & $12.40(7.37)$ & $9.81(5.92)$ & $0.025 * *$ & $8.81(5.46)$ & $12.64(6.53)$ & 0.065 \\
\hline GSI score at SCL-90R* & $15.88(9.38)$ & $12.85(9.14)$ & $0.031 * *$ & $12.87(9.63)$ & $12.14(7.91)$ & 0.825 \\
\hline SDS score* & $8.59(2.14)$ & $5.68(3.20)$ & $0.000 * *$ & $4.39(2.35)$ & $9.36(2.06)$ & $0.000 * *$ \\
\hline
\end{tabular}

* Valued expressed as Mean (SD)

** Correlation is significant at the 0.01 level (2-tailed)

Specifically, we decided to test some clinical and affective variables known to predict relapse after a successful detoxification. We did not find risk factors for any of the three selected outcomes at 1-year follow-up (the presence of relapse, the headache-related disability and the number of tablets per month). We hypothesize that detoxification brings improvements in most of the clinical and affective measures, but does not free these severely affected migraineurs from significant pain intensity, headacherelated disability and dependency-like behaviors, which place them at risk for new chronicity and recurrent relapses. The partial benefit from detoxification and the severity bias led us to consider this subgroup of chronic-relapsing migraneurs as a separate phenotype, whose features might belong to the substance use disorders spectrum. Recent findings from neuroimaging and neuropsychological studies give strength to this hypothesis $[6-8,30]$. However, this study has a number of limitations. First, due to the high proportion of dropouts (a further indicator of severity within our sample), we could not determine whether these patients would have had a worse clinical and affective profile. Second, the sample size might account for the lack, the limited reliability and the exploratory nature of these preliminary findings. Third, the considered variables, as shown by Pearson analysis, went through a degree of reciprocal influences that could affect the attempt of determining the single predicting value.

Further studies are needed to design specific management protocols for migraine according to the medication overuse severity. Since we believe changes in pain locus of control fundamental for treatment outcomes, we suggest a multidisciplinary therapeutic program for $\mathrm{CM}$ patients with MO.
Conflict of interest I certify that there is no actual or potential conflict of interest in relation to this article.

\section{References}

1. Bigal ME, Rapoport AM, Sheftell FD, Tepper SJ, Lipton RB (2004) Transformed migraine and medication overuse in a tertiary headache centre. Clinical characteristics and treatment outcomes. Cephalalgia 24:483-490

2. Diener HC, Katsarava Z, Limmroth V (2010) Headache attributed to a substance or its withdrawal. Handb Clin Neurol 97:589-599

3. Evers S, Marziniak M (2010) Clinical features, pathophysiology and treatment of medication-overuse headache. Lancet Neurol 9(4):391-401

4. Radat F, Lanteri-Minet M (2010) What is the role of dependencerelated behavior in medication-overuse headache? Headache 50(10):1597-1611

5. Andrasik F, Grazzi L, Usai S, D’Amico D, Kass S, Bussone G (2007) Disability in chronic migraine with medication overuse: treatment effects at 3 years. Headache 47(9):1277-1281

6. Fumal A, Luareys S, Di Clemente L et al (2006) Orbitofrontal cortex involvement in chronic analgesic-overuse headache evolving from episodic migraine. Brain 129(2):543-550

7. Mongini F, Keller R, Deregibus A, Barbalonga E, Mongini T (2005) Frontal lobe dysfunction in patients with chronic migraine: a clinical-neuropsychological study. Psychiatry Res 133(1):101-106

8. Gòmez-Beldarrain M, Carrasco M, Bilbao A, Garcìa-Moncò JC (2011) Orbitofrontal dysfunction predicts poor prognosis in chronic migraine with medication overuse. J Headache Pain 12(4):459-466

9. Lake AE, Jr Saper, Hamel RL (2009) Comprehensive inpatient treatment of refractory chronic daily headache. Headache 49:555-562

10. Grazzi L, Chiapparini L, Ferraro S, Usai S, Andrasik F, Mandelli ML, Bruzzone MG, Bussone G et al (2010) Chronic migraine with medication overuse pre-post withdrawal of symptomatic medication: clinical results and fMRI correlations. Headache 50(6):998-1004 
11. Katsarava Z, Muessig M, Dzagnidze A, Fritsche G, Diener HC, Limmroth V (2004) Medication overuse headache: rates and predictors for relapse in a 4-year prospective study. Cephalalgia 25:12-15

12. Rossi R, Faroni JV, Nappi G (2008) Medication overuse headache: predictors and rates of relapse in migraine patients with low medical needs. A 1-year prospective study. Cephalalgia 28: 1196-1200

13. Mongini F, Keller R, Deregibus A, Raviola F, Mongini T, Sancarlo M (2003) Personality traits, depression and migraine in women. A longitudinal Study. Cephalalgia 23:186-192

14. Austin MP, Ross M, Murray C, O'Carroll RE, Ebmeier KP, Gm Goodwin (1992) Cognitive function in major depression. J Affect Disord 25:21-29

15. Breslau N, Davis GC, Schultz LR, Peterson EL (1994) Migraine and major depression: a longitudinal study. Headache 34: 387-393

16. Grande RB et al (2009) The Severity of Dependence Scale detects people with medication overuse: the Akershus study of chronic headache. Neurol Neurosurg Psychiatry 80(7):784-789

17. Lundqvist $C$ et al (2011) An adapted Severity of Dependence Scale is valid for the detection of medication overuse: the Akershus study of chronic headache. Eur J Neurol 18(3):512-518

18. Lundqvist $\mathrm{C}$ et al (2010) The severity of dependence score correlates with medication overuse in persons with secondary chronic headaches. The Akershus study of chronic headache. Pain 148(3):487-491

19. Lauwerier E, Paemeleire K, Van Damme S, Goubert L, Crombez G (2011) Medication use in patients with migraine and medication-overuse headache: the role of problem-solving and attitudes about pain medication. Pain 152:1334-1339

20. Zidverc-Trajkovic J, Pekmezovic T, Jovanovic Z, Pavlovic A, Mijajlovic M, Radojicic A et al (2007) Medication overuse headache: clinical features predicting treatment outcome at 1-year follow-up. Cephalalgia 27:1219-1225

21. Hagen K, Albretsen C, Vilming ST, Salvesen R, Grønning M et al (2011) A 4-year follow-up of patients with medication-overuse headache previously included in a randomized multicentre study. J Headache Pain 12:315-322

22. Bøe MG, Salvesen R, Mygland A (2009) Chronic daily headache with medication overuse: predictors of outcome 1 year after withdrawal therapy. Eur J Neurol 16:705-712

23. Silberstein SD, Olesen J, Bousser MG et al (2005) The International Classification of Headache Disorders, 2nd edition (ICHDII)-revision of criteria for 8.2 Medication-overuse headache. Cephalalgia 25:460-465

24. Hamilton M (1967) Development of a rating scale for primary depressive illness. Br J Soc Clin Psychol 6:278-296

25. Hamilton M (1959) The assessment of anxiety states by rating. $\mathrm{Br}$ J Med Psychol 32:50-55

26. Stewart WF et al (2001) Development and testing of the Migraine Disability Assessment (MIDAS) Questionnaire to assess headache-related disability. Neurology 56(6 Suppl 1):S20-S28

27. D'Amico D, Mosconi P, Genco S, Usai S, Prudenzano AM, Grazzi L, Leone M, Puca FM, Bussone G (2001) The Migraine Disability Assessment (MIDAS) questionnaire: translation and reliability of the Italian version. Cephalalgia 21(10):947-952

28. Gossop M et al (1997) Test-retest reliability of the Severity of Dependence Scale. Addiction 92(3):353

29. Hardt J, Gerbershagen HU, Franke P (2000) The symptom checklist, SCL-90-R: its use and characteristics in chronic pain patients. EurJ Pain 4(2):137-148

30. Biagianti B, Grazzi L, Gambini O, Usai S, Muffatti R, Scarone S, Bussone G (2012) Orbitofrontal dysfunction and medication overuse in patients with migraine. Headache. doi: 10.1111/j. 1526-4610.2012.02277.x [Epub ahead of print] 